\title{
Efficient and Clean Catalytic Hydrogenolysis of Aromatic Ketones by Silica Supported Schiff Base Modify Chitosan-Palladium Catalyst
}

\author{
Tingting Gu, Lijun Liu*, Changqiu Zhao \\ Shandong Provincial Key Laboratory of Chemical Energy-Storge and Novel Cell Technology, \\ School of Chemistry and Chemical Engineering, Liaocheng University, Liaocheng, China \\ Email: "gongshw@1cu.edu.cn
}

Received November 14, 2012; revised December 19, 2012; accepted December 28, 2012

\begin{abstract}
An silica supported chitosan-Schiff base Pd(II) catalyst was prepared in a simple way and characterized by XRD, FT-IR, SEM-EDS, XPS and TG, and the ability of this complex to catalyze hydrogenolysis of 1-tetralone into 1,2,3,4-tetrahydronaphthalene was also investigated in the presence of hydrogen. It has been revealed that the catalyst had high catalytic activity for hydrogenolysis of 1-tetralone at ambient temperature and normal pressure of hydrogen. Especially, the hydrogenolysis of 1-tetralone in ethanol solvent gave excellent results and the $100 \%$ conversion of 1-tetralone and the $100 \%$ selectivity for 1,2,3,4-tetrahydronaphthalene were obtained under optimized reaction conditions. The influences of reaction temperature, reaction time and solvent on the hydrogenolysis of 1-tetralone were also investigated. It has been also revealed that the catalyst was efficient and eco-friendly for the hydrogenolysis of carbonyl that connected with a benzene ring to give corresponding aromatic hydrocarbons.
\end{abstract}

Keywords: CS-Schiff-Base; Pd Catalyst; Hydrogen; Aromatic Ketones

\section{Introduction}

The reduction of aldehydes or ketones, especially selectively reduce $\mathrm{C}=\mathrm{O}$ group to methylene $\left(\mathrm{CH}_{2}\right)$ is an important organic reaction, which is used to the direct conversion of aromatic ketones to synthesize linear alkylbenzenes [1]. The linear alkylbenzenes are frequently used as intermediates in chemical industries. The classical reported procedures for the reduction of $\mathrm{C}=\mathrm{O}$ group to $\mathrm{CH}_{2}$ are the Clemmensen and Wolff-Kishner reducetions [2,3]. However, not only Clemens reaction, but also Wolff-Kishner reaction is not environment-friendly, these reactions are either carried out in the presence of $\mathrm{Zn}-\mathrm{Hg} /$ concentrated $\mathrm{HCl}$ or employed lot of hydrazine as reactant. Subsequently, the catalytic hydrogenation of $\mathrm{C}=\mathrm{O}$ group to $\mathrm{CH}_{2}$ has been reported, $\mathrm{Cu}-\mathrm{Cr}, \mathrm{Fe}$ or $\mathrm{Ni}$ had proved less active for the conversion of $\mathrm{C}=\mathrm{O}$ into $\mathrm{CH}_{2}$ at high temperatures (473-573 K) [4-6]. Avnir [7] once reported that the catalytic hydrogenolysis of aromatic ketones by a sol-gel entrapped combined Pd$[\mathrm{Rh}(\mathrm{cod}) \mathrm{Cl}]_{2}$ catalyst, however, the selectivity of hydrogenation reaction towards the alkylbenzenes is low and the aromatic rings were fully hydrogenated. The result that reported by Prof. De Vos [8] suggests that the mixed

"Corresponding author. choline-betainium ionic liquids has promotional effect on the hydrogenolysis of aromatic ketones catalytic by Pd catalyst, the conversion of aromatic ketones and selectivity to alkylbenzenes were all improved.

Chitosan (CS) is a natural biopolymer, which can be easily obtained from chitin that is widely dispersed in living organisms [9]. More recently, with the development of environmentally friendly industries, CS-supported metals catalysts have attracted a lot of attention because CS shows these advantages of nontoxicity and desirable physical and mechanical properties [10-13]. In previous papers [14-18], a silica-supported CS-palladium complex abbreviated as SiO-CS-Pd has been found to catalyze the hydrogenation of nitrotoluene, nitrobenzene, chlorophenol, 2-octene and 3-octene, et al. Some ketones can reduce to chiral alcohols catalyzed by silica supported CS-Pd complex via asymmetric hydrogenation $[19,20]$. However, the successful application of CS stabilized palladium catalysts in hydrogenolysis reactions of $\mathrm{C}=\mathrm{O}$ to $\mathrm{CH}_{2}$ groups has not yet been reported.

In the presence of active amino group, CS also exhibits the possibility of chemical modifications, including the preparation of Schiff bases by reaction with aldehydes and ketones [21-23]. Recently, we has prepared a silica supported Schiff base modified CS-Pd catalyst and 
found that it exhibited good catalytic activity in the hydrogenaolysis of 1-tetralone. In this paper, we validated this finding and revealed the application scope of the catalyst. It can be found that this immobilized combined catalyst promotes the total hydrogenolysis of some aromatic ketones to give corresponding aromatic hydrocarbons under mild conditions.

\section{Experimental}

\subsection{Materials}

Chitosan (CS) finely purified to a de-acetyl degree of 90.0\% was purchased from Sinopharm Chemical Reagent Co, Ltd. China. Salicylaldehyde and 1-tetralone was purchased from Aladdin Reagent Co, Ltd. China and salicylaldehyde was distilled before used. Other reagents were of analytical grade and were used as received.

\subsection{Preparation of Catalyst}

Silica supported chitosan $\left(\mathrm{SiO}_{2}-\mathrm{CS}\right)$ was first prepared, $4.0 \mathrm{~g} \mathrm{CS}$ was added into $250 \mathrm{ml} 1.5 \% \mathrm{CH}_{3} \mathrm{COOH}$ and stirred until $\mathrm{CS}$ was all dissolved at room temperature, and then $8.0 \mathrm{~g} \mathrm{SiO}_{2}$ was added. After continued stirred for $2 \mathrm{~h}$, the $\mathrm{PH}$ of solution was modified by $1 \mathrm{~mol} / \mathrm{L}$ $\mathrm{NaOH}$ to 13 , the solid was separated by filtration, washed with water (until the $\mathrm{PH}=8$ ), ethanol and acetone, respectively, and then dried at $333 \mathrm{~K}$ under vacuum for $10 \mathrm{~h}$ to give white solid, the nitrogen content was determined to be $2.52 \mathrm{wt} \%$ by elemental analysis.

Then the silica supported chitosan Schiff base ( $\mathrm{SiO}_{2}-\mathrm{CS}-\mathrm{Schiff}$ base) was prepared refer to the procedure in the literatures $24-25.10 \mathrm{~g} \mathrm{SiO}_{2}-\mathrm{CS}$ and excess salicyal $(15 \mathrm{ml})$ and acetic acid $(12 \mathrm{ml})$ were added to methanol $(120 \mathrm{ml})$, and then the mixture was refluxed for $10 \mathrm{~h}$. After the resultant mixture was cooled, the solid was separated by filtration, washed with methanol and then dried at $333 \mathrm{~K}$ under vacuum for $12 \mathrm{~h}$ to give bright yellow solid.

Finally, the silica supported Schiff base modify chitosan-palladium $\left(\mathrm{SiO}_{2}-\mathrm{CS}-\mathrm{Schiff}\right.$ base-Pd) was prepared according to the method of reported in literatures $[18,19$, 23]. The $\mathrm{SiO}_{2}-\mathrm{CS}$ Schiff-base and $\mathrm{PdCl}_{2}$ were weighed in 10:1 mass ratio and were added in ethanol solvent. After the mixture was stirred at $303 \mathrm{~K}$ for $72 \mathrm{~h}$, the solid product was filtered and washed with ethanol to the filtrate became transparent and colorless, and dried at $323 \mathrm{~K}$ under vacuum to obtained brown catalyst particles, which was then used to catalyze the hydrogenolysis of 1-tetralone with hydrogen. The metal contents of catalyst determined by ICP are $5.4 \%$.

\subsection{Characterization of the Catalyst}

The X-ray diffraction analysis was carried out using a
X-ray diffractometer (Beijing Purkinje General Instrument Co. Ltd) with $\mathrm{Ni}$ filtered $\mathrm{Cu} \mathrm{K}_{\alpha}$ radiation $(\lambda=1.542$ $\AA$ ) and a scanning range $2 \theta$ of $5^{\circ}-80^{\circ}$. The FT-IR spectra were measured on a FT6700 spectrophotometer in the range $400-4000 \mathrm{~cm}^{-1}$. X-Ray Photoelectron Spectroscopy (XPS) measurements were performed with a VG Scientific ESCALAB 250 instrument with $\mathrm{Mg} \mathrm{K} \alpha$ radiation $(1253.6 \mathrm{eV})$. The TG analyses were performed on a STA449 thermogravimetric analyzer (NETZSCH, Germany). The morphology and elemental composition of sample was analyzed by a JSM6380LV scanning electron microscopy equipped with energy dispersive X-ray (SEM-EDX) elemental analysis system. The content of Pd before and after reaction was identified by an OPTIMA2000DV Inductive Coupled Plasma Emission Spectrometer (ICP).

\subsection{Hydrogenolysis of 1-Tetralone}

The hydrogenolysis experiment was performed in a 50 $\mathrm{mL}$ tube with a side neck equipped with a magnetic stirrer and an automatic temperature controller. In a typical hydrogenolysis experiment, to a mixture of 1-tetralone substrates $(0.5 \mathrm{ml})$ and catalyst $(0.2 \mathrm{~g})$ was added ethanol $(15 \mathrm{ml})$. The reaction vessel was flushed (three times) with pure hydrogen and then retained the $40 \mathrm{ml} / \mathrm{min}$ current velocity of hydrogen. The reaction mixture was stirred magnetically at a rate of $150 \mathrm{rpm}$ at $313 \mathrm{~K}$ for $5 \mathrm{~h}$. After the reaction, the hydrogenolysis products were identified and quantified by ${ }^{1} \mathrm{H} \mathrm{NMR}\left(\mathrm{CDCl}_{3}, 400 \mathrm{MHz}\right)$ and an HP 6890/5973 GC/MS instrument (FID, column: HP-5MS). The by-products of the reaction were 1-tetralin alcohol.

\section{Results and discussion}

\subsection{Characterization Results}

The XRD pattern of prepared catalyst is shown in Figure 1. From the pattern, it can be seen that $\mathrm{SiO}_{2}-\mathrm{CS}$-Schiffbase-Pd only exhibits two broad peaks at about $20^{\circ}$, which ascribes the amorphous silica and the crystalline macromolecule of $\mathrm{CS}$, respectively. The diffraction peaks of palladium (0) $\left(2 \theta=38^{\circ}, 46^{\circ}\right.$, et al., JCPDS: 05-618) or other palladium-contained compounds are not observed.

The FT-IR spectrums of the prepared $\mathrm{SiO}_{2}$-CS-Schiffbase and $\mathrm{SiO}_{2}-\mathrm{CS}-\mathrm{Schiff}$-base-Pd catalyst had reported in previous works [25]. The results exhibited that a band due to the $\mathrm{C}=\mathrm{N}$ stretching vibration appeared at 1630 $\mathrm{cm}^{-1}$, which indicated that the formation of Schiff base And the characters bands of silica were also retained, which means that the structure of silica was no change in the preparation.

The TG data of the $\mathrm{SiO}_{2}-\mathrm{CS}$-Schiff-base-Pd catalyst is depicted in Figure 2. From this result, the destruction 


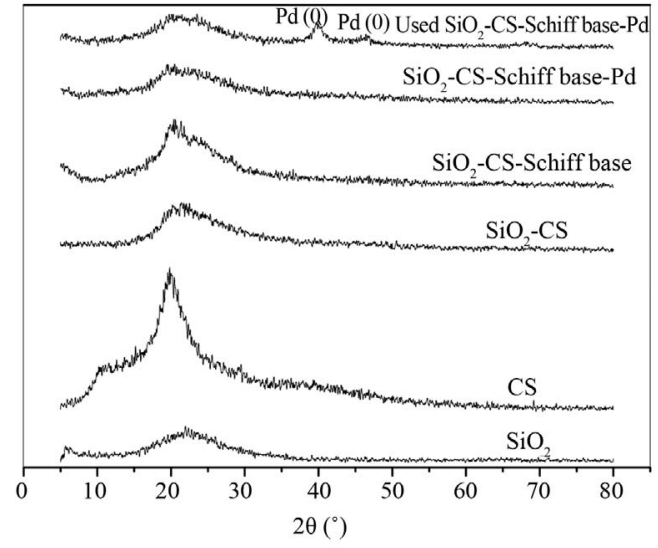

Figure 1. XRD pattern of silica supported CS-Schiff-basePd catalyst.

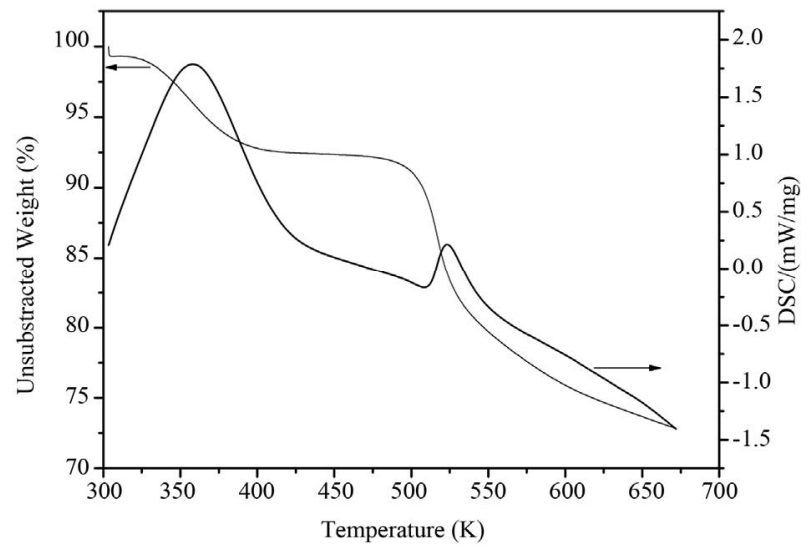

Figure 2. The TG data of silica supported CS-Schiff-basePd catalyst.

temperature of catalyst is $490 \mathrm{~K}$, which suggests that the catalyst of $\mathrm{SiO}_{2}$-CS-Schiff-base-Pd have a good thermal stability between room temperature and $490 \mathrm{~K}$. The weight loss of the catalysts before the destruction is attributed to desorption of water.

The surface morphology of the catalyst is shown in Figure 3. From the SEM image it is clear that the catalyst has irregular block structure. And the existence of palladium in the catalyst was confirmed by SEM-EDX analysis (Figure 3), chloride also was detected for $\mathrm{SiO}_{2}-$ CS-Schiff-base-Pd catalyst. The elemental composition of catalyst analyzed by with SEM-EDX is listed in Table 1, and it can be seen that the molar ratio of chlorine: palladium is about $2: 1$. The appearance of the $\mathrm{SiO}_{2}-\mathrm{CS}$ Schiff-base-Pd catalyst and $\mathrm{SiO}_{2}$-CS-Schiff-base is different in color. The former was brown and the latter was bright yellow.

Figure 4 shows the XPS analysis of $\mathrm{SiO}_{2}-\mathrm{CS}-\mathrm{Schiff}-$ base-Pd catalyst. The XPS analysis is a technique essentially limited to the very first external layers of the material (on a thickness limited to 2 - 3 times the wavelength

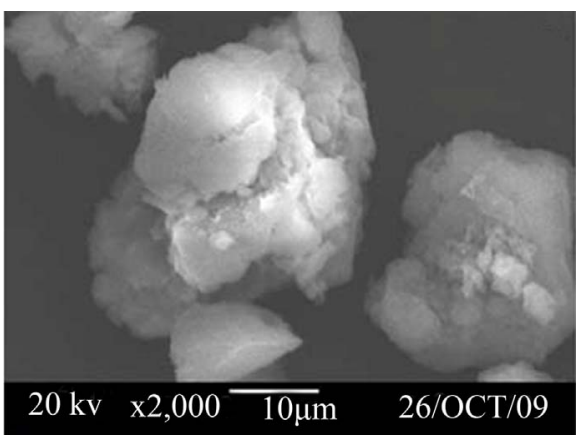

(a)

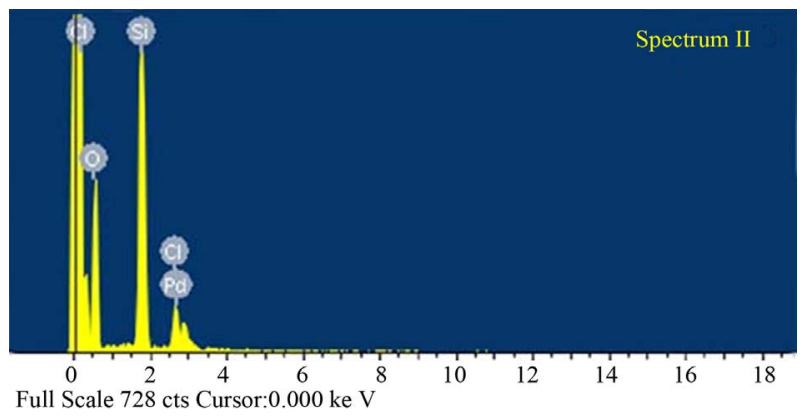

(b)

Figure 3. (a) SEM image; (b) SEM-EDS of silica supported CS-Schiff-base-Pd catalyst.

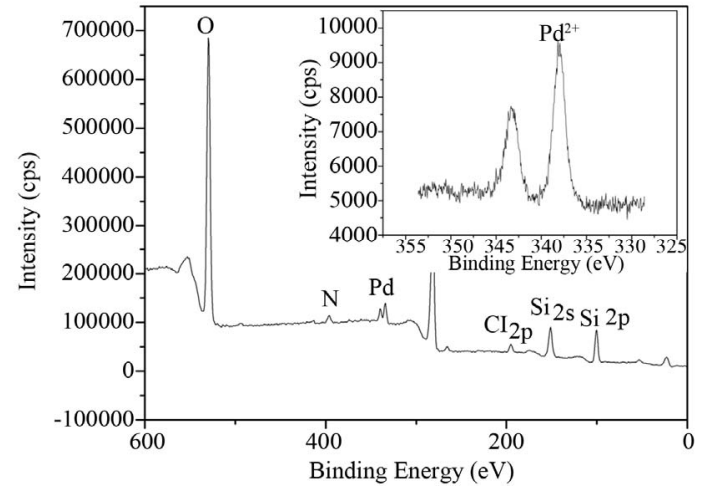

Figure 4. XPS analysis of SiO2-CS-Schiff-base-Pd catalyst.

Table 1. The element content and Surface area of $\mathrm{SiO}_{2}-\mathrm{CS}$ Schiff-base-Pd catalyst.

\begin{tabular}{|c|c|c|c|c|c|}
\hline & \multicolumn{4}{|c|}{$\begin{array}{l}\text { Elemental Composition }{ }^{\mathrm{a}} \\
(\mathrm{mol} \%)\end{array}$} & \multirow{2}{*}{$\begin{array}{l}\text { BET Surface } \\
\operatorname{area}\left(\mathrm{m}^{2} \cdot \mathrm{g}^{-1}\right)\end{array}$} \\
\hline & $\mathrm{O}$ & $\mathrm{Si}$ & $\mathrm{Cl}$ & $\mathrm{Pd}$ & \\
\hline $\mathrm{SiO}_{2}$ & - & - & - & - & 380 \\
\hline $\mathrm{SiO}_{2}$-CS-Schiff base-Pd & 78.44 & 17.83 & 2.50 & 1.23 & 68 \\
\hline $\begin{array}{c}\text { Used } \mathrm{SiO}_{2}-\mathrm{CS}-\mathrm{Schiff} \\
\text { base-Pd }\end{array}$ & 68.54 & 26.04 & 3.57 & 1.85 & 64 \\
\hline
\end{tabular}

${ }^{\mathrm{a}}$ Analyzed by SEM-EDX.

of the analytical radiation) and the analysis result confirms the existence of $\mathrm{O}, \mathrm{N}, \mathrm{Si}, \mathrm{Cl}$ and $\mathrm{Pd}$. One peak was 
identified at $337.6 \mathrm{eV}$, corresponding to the $\mathrm{Pd}^{2+}$ forms, which means that the $\mathrm{Pd}^{2+}$ is the main existence of $\mathrm{Pd}$ in the prepared catalyst. The result is consistent with the analysis of SEM-EDX (Table 1) that the elemental composition of $\mathrm{Cl}$ is close to two times that of $\mathrm{Pd}$.

\subsection{Catalytic Activity}

Catalytic activity of the $\mathrm{SiO}_{2}$-CS-Schiff-base-Pd catalyst was evaluated for the hydrogenolysis of 1-tetralone in the presence of $\mathrm{H}_{2}$ and ethanol. For comparison, CS-Pd, CS-Schiff base-Pd, ZSM and $\mathrm{Al}_{2} \mathrm{O}_{3}$ supported CS-Schiff base-Pd were prepared and also examined as hydrogenolysis catalyst with same conditions, and the results are showed in Table 2. As showed in Table 2, there is no reaction took place in the absence of catalyst or in the presence of CS-Pd. After $3 \mathrm{~h}$ hydrogenation, $\mathrm{SiO}_{2}$-CS-Pd exhibited activity of hydrogenation but only 1-tetrahydronaphthalenol was formed as the sole product, which was accordance with reported results that silica supported CS-Pd catalyst has catalytic activity for hydrogenation of ketones to form corresponding alcohols $[19,20]$. Yuan [19] once reported that the asymmetry transfer hydrogenation of acetophenone catalyzed by silica supported CS-Pd catalyst, and an optical yield of $R$-1-phenylethanol could reach $99 \%$ after $8 \mathrm{~h}$ reaction at $303 \mathrm{~K}$ under atmosphere hydrogen. In the presence of unsupported CS-Schiff base-Pd catalyst, 1-tetralone totally converted and the expected tetrahydronaphthalene in the yield of $37 \%$ and 1-tetrahydronaphthalenol in the yield of $63 \%$. In this sense the modification of chitosan with salicylaldehyde to produce Schiff base can improve the hydrogenation selectivity of catalyst via the coordination between chitosan and palladium atom, in other words, the insertion of functional group of Schiff base in the chitosan matrix may improve its capacity of interaction with metallic ions by complexation [24]. For supported catalyst, the $\mathrm{SiO}_{2}$-CS-Schiff-base-Pd, gave $100 \%$ conversion of 1-tetralone and tetrahydronaphthalene formed by $72 \%$ yield, proved to be the most efficient for hydrogenolysis, which suggests that silica is an excellent support to optimize the catalytic activity and selectivity, which may be due to the high specific surface area of silica to enhance the catalytic chance of substrates and active sites of catalyst. Therefore, $\mathrm{SiO}_{2}$-CS-Schiff-base-Pd was chosen as catalyst for full reduction of $\mathrm{C}=\mathrm{O}$ to $\mathrm{CH}_{2}$, and the effect of reaction parameters like temperature, the catalyst amount and the solvent on the conversion of 1-tetralone and selectivity to tetrahydronaphthalene has been investtigated in detail.

\subsubsection{The effect of Reaction Temperature}

As shown in Figure 5, the conversion increased to the maximum with the rise in the reaction temperature, and $69 \%$ of 1-tetralone conversed at room temperature, which suggests that the $\mathrm{SiO}_{2}$-CS-Schiff base-Pd catalyst exhibited excellent hydrogenation activity even at low temperature. 1-tetralone totally conversed when temperature was raised to $303 \mathrm{~K}$. The selectivity for tetrahydronaphthalene and 1-tetrahydronaphthalenol as a function of the reaction temperature are also shown in Figure 6. It is clear that high temperature favors the formation of tetrahydronaphthalene and 1-tetralone all transformed into tetrahydronaphthalene at $313 \mathrm{~K}$.

\subsubsection{The Effect of Reaction Time}

Figure 6 presents the effects of reaction time on 1-tetralone hydrogenolysis with hydrogen in ethanol. The reaction hardly occurred during the first $0.5 \mathrm{~h}$, which might be the initiation period. After the initiation period, the 1-tetralone conversion increased as the reaction time was prolonged and the $100 \%$ conversion was obtained after 2 $\mathrm{h}$ reaction. In this period, the selectivity of tetrahydronaphthalene has slight decrease with the extension of time, and then increases with further prolonging the reaction time, $100 \%$ 1-tetralone was reduced to tetrahydronaphthalene after $4 \mathrm{~h}$ reaction. This result suggests that 1-tetralone may firstly converses to 1-tetrahydronaphthalenol by hydrogenation, and then tetrahydronaphthalene

Table 2. Hydrogenolysis of 1-tetralone catalyzed by different catalysts.

\begin{tabular}{cccc}
\hline \multirow{2}{*}{ Catalyst } & Conversion $(\%)$ & \multicolumn{3}{c}{ Selectivity (\%) } \\
\cline { 3 - 5 } No & - & tetrahydronaphthalene & 1-tetrahydronaphthalenol \\
CS-Pd & - & - & - \\
$\mathrm{SiO}_{2}$-CS-Pd & 36 & - & 100 \\
CS-Schiff base-Pd & 100 & 37 & 63 \\
$\mathrm{ZSM}_{2}$-CS-Schiff base-Pd & 100 & 25 & 75 \\
$\mathrm{Al}_{3} \mathrm{O}_{2}$-CS-Schiff base-Pd & 100 & 36 & 64 \\
$\mathrm{SiO}_{2}$-CS-Schiff base-Pd & 100 & 72 & 28
\end{tabular}

${ }^{\mathrm{a}}$ Reaction conditions: $0.5 \mathrm{~mL}$ substrate, $0.2 \mathrm{~g}$ catalyst, $15 \mathrm{~mL}$ ethanol, $40 \mathrm{~mL} / \mathrm{min} \mathrm{H}_{2}, 313 \mathrm{~K}$. 


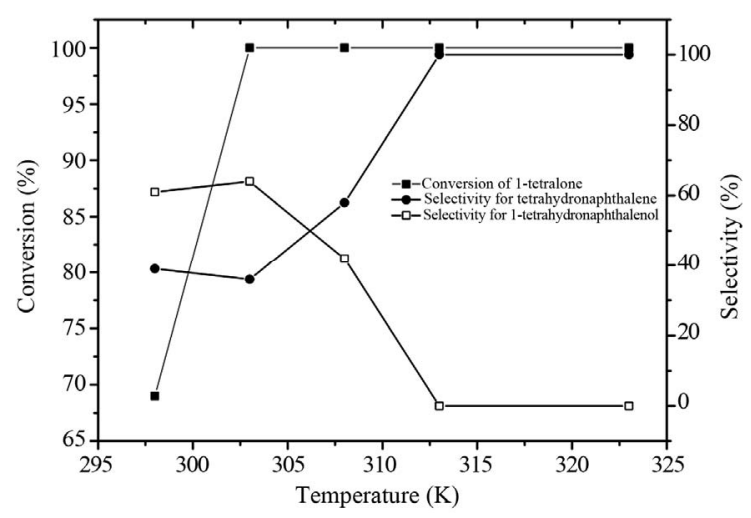

Figure 5. Effect of reaction temperature on deoxygenation of 1-tetralone.

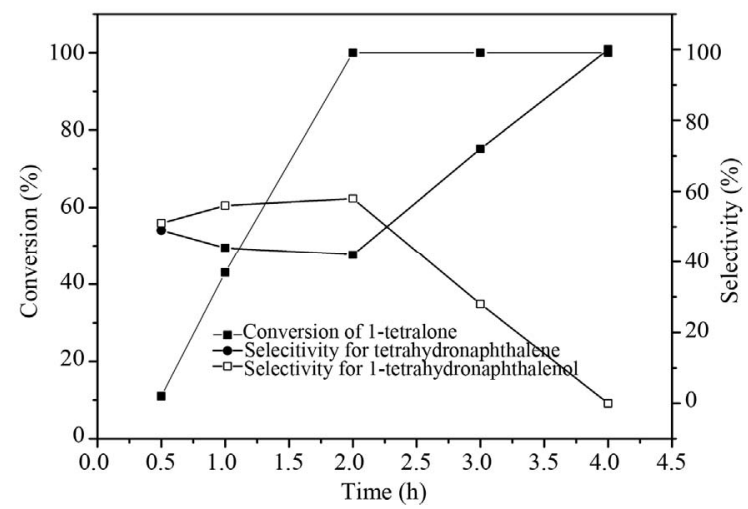

Figure 6. Effect of reaction time on deoxygenation of 1tetralone.

is formed by hydrogenolysis after a prolonged reaction period.

\subsubsection{Effect of Catalyst Amount}

The effect of catalyst amount on conversion and selectivity was examined by varying the catalyst amount from 0.05 to $0.25 \mathrm{~g}$ (Figure 7). The conversion of 1-tetralone was observed to be only $18 \%$ with $27 \%$ selectivity to tetrahydronaphthalene for a $0.05 \mathrm{~g}$ amount of catalyst. The conversion of 1-tetralonn increased to $100 \%$ at a $0.15 \mathrm{~g}$ of the catalyst amount with $53 \%$ selectivity to tetrahydronaphthalene. The selectivity to tetrahydronaphthalene also increased to be $100 \%$ when $0.2 \mathrm{~g}$ catalyst was used, and no significant change in the conversion and selectivity was observed on a further increase in the catalyst amount. With increasing of catalyst amount, the conversion and selectivity to tetrahydronaphthalene all increased, which may be explained basis that the increase of adsorption sites with the increasing of catalyst amount. And it can be clear found that the totally conversion was first obtained and then the $100 \%$ selectivity to hydrogenolysis was obtained, which definitelysuggests that 1-tetrahydronaphthalenol shall further transform into tetrahydronaphthalene by hydrogenolysis.

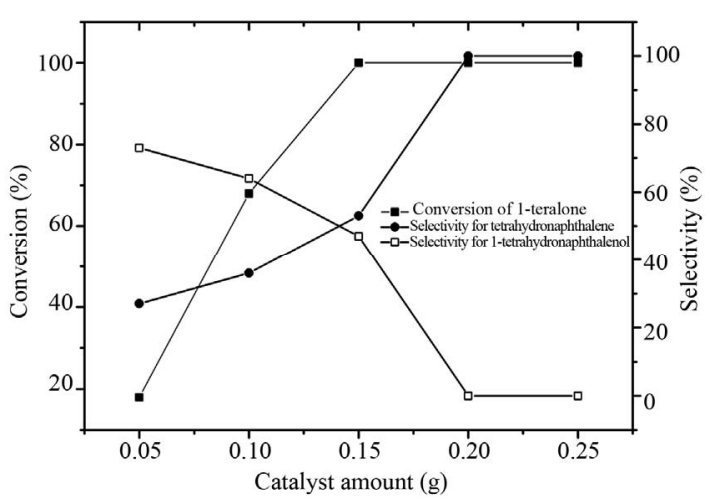

Figure 7. Effect of catalyst amount on deoxygenation of 1tetralone.

\subsubsection{Effect of Solvent}

Different solvents were studied for $\mathrm{SiO}_{2}$-CS-Schiff basePd catalyzed hydrogenolysis of 1-tetralone. As showed in Table 3, proton solvents were essential to the reaction. In ethanol or methanol, the reaction gave $100 \%$ conversion but by-product 1-tetrahydronaphthalenol formed by $39 \%$ when the reaction was carried out in methanol. In acetonitrile, although the $100 \%$ conversion was obtained, the main reduced product was 1-tetrahydronaphthalenol and only $11 \%$ 1-tetralone was conversed into tetrahydronaphthalene. In cyclohexane, benzene, carbon tetrachloride or in the absence of solvent, the reaction hardly occurs or cannot take place.

\subsection{Catalyst Recycling}

In order to observe the recycle ability of the catalyst, the hydrogenolysis reaction was performed with $0.2 \mathrm{~g}$ of the catalyst at $313 \mathrm{~K}$ in ethanol and the results are given in Table 4. For the fresh catalyst the conversion and selectivity to tetrahydronaphthalene were all $100 \%$ after $4 \mathrm{~h}$ reaction. The filtered catalyst was only dried at $323 \mathrm{~K}$ under vacuum and reused under identical conditions with the fresh charge of other materials. There is a decrease in conversion and selectivity to tetrahydronaphthalene with the increase of reused times, after used three times, catalyst gave only $57 \%$ conversion and $37 \%$ selectivity to tetrahydronaphthalene. However, the extension of reaction time was still favor the increase of conversion and selectivity to tetrahydronaphthalene, for second recycle catalyst, the total conversion and $81 \%$ selectivity to tetrahydronaphthalene was gave after $6 \mathrm{~h}$ reaction.

The third recycle catalyst was analyzed by ICP, the analysis result suggests the content of Pd increased to $6.6 \%$ from $5.4 \%$ of the fresh catalyst, and the similar result can be observed basis on the analysis result of SEM-EDX (Table 1), too. The increase of content of Pd may be due to the loss or the decomposition of CS and the functional group of Schiff base in the polarity reaction condition, and then results in the decrease of cata- 
Table 3. Effect of solvent on hydrogenolysis of 1-tetralone.

\begin{tabular}{cccc}
\hline Solvent & Conversion (\%) & \multicolumn{2}{c}{ Selectivity (\%) } \\
\cline { 3 - 4 } & & tetrahydronaphthalene & 1-tetrahydronaphthalenol \\
\hline Neat & - & - & - \\
Ethanol & 100 & 100 & 39 \\
Methanol & 100 & 11 & 89 \\
Acetonitrile & 100 & 100 & 0 \\
Cyclohexane & 4 & 100 & 0 \\
Benzene & 2 & - & - \\
Carbon tetrachloride & - &
\end{tabular}

Reaction conditions: $0.5 \mathrm{~mL}$ substrate, $0.2 \mathrm{~g}$ catalyst, $15 \mathrm{~mL}$ solvent, $313 \mathrm{~K}, 40 \mathrm{~mL} / \mathrm{min}_{2}, 4 \mathrm{~h}$.

Table 4. Effect of recycle of the catalyst on hydrogenolysis of 1-tetralone.

\begin{tabular}{cccc}
\hline \multirow{2}{*}{ Used times } & Conversion $(\%)$ & \multicolumn{2}{c}{ Selectivity (\%) } \\
\cline { 3 - 4 } & 100 & tetrahydronaphthalene & 1-tetrahydronaphthalenol \\
\hline 1 & 100 & 100 & 0 \\
2 & 57 & 36 & 63 \\
$3^{\mathrm{a}}$ & 100 & 37 & 19 \\
4 & 34 & 81 & 71 \\
\hline
\end{tabular}

Reaction conditions: $0.5 \mathrm{~mL}$ substrate, $0.2 \mathrm{~g}$ catalyst, $15 \mathrm{~mL}$ ethanol, $313 \mathrm{~K}, 40 \mathrm{~mL} / \mathrm{min}_{2}, 4 \mathrm{~h}{ }^{\mathrm{a}}$ Result was obtained after $6 \mathrm{~h}$ reaction.

lytic activity in hydrogenolysis reaction. The XRD pattern of the third recycle catalyst clearly exhibits two diffraction peaks at $40.5^{\circ}$ and $46.5^{\circ}$, which indicates the presence of $\operatorname{Pd}(111)$ and $\operatorname{Pd}(110)$ phase which are attributed to $\operatorname{Pd}(0)$ (Figure 1), which indicates that partially $\mathrm{Pd}$ (II) was reduced to $\mathrm{Pd}(0)$.

\subsection{Extension to Other Substrates}

To deep reveal the catalytic hydrogenolysis activity of $\mathrm{SiO}_{2}-\mathrm{CS}-\mathrm{Schiff}$ base-Pd catalyst, some hydrogenation experiments of representative carbonyl compounds were carried out and these results are summarized in Table 5. In the presence of $\mathrm{SiO}_{2}-\mathrm{CS}-\mathrm{Schiff}$ base-Pd catalyst, the hydrogenation of examined aliphatic did not occur besides cyclohexanone (entry 1 - 4), and only $42 \%$ cyclohexanone was reduced to cyclohexanol, which suggests that the $\mathrm{SiO}_{2}-\mathrm{CS}$-Schiff base-Pd catalyst is inactive for hydrogenation of aliphatic ketones. For examined aromatic carbonyl compounds, the dexoygenation of benzaldehyde (entry 5), acetophenone (entry 6) and propiophenone (entry 7 ) gave $100 \%$ conversion and $100 \%$ selectivity to corresponding aromatic hydrocarbon, which means that the $\mathrm{C}=\mathrm{O}$ was totally transformed into $\mathrm{CH}_{2}$, in other words, $\mathrm{SiO}_{2}$-CS-Schiff base-Pd catalyst has excellent catalytic activity for hydrogenolysis of aromatic aldehyde or ketones. When the electron-donating group, such as $\mathrm{OCH}_{3}$ and $\left(\mathrm{CH}_{3}\right)_{2} \mathrm{~N}$ was introduced to benzene ring (entry 8 - 9), the $\mathrm{C}=\mathrm{O}$ was also effectively reduced to $\mathrm{CH}_{2}$. When the electron-withdrawing group, such as $\mathrm{Cl}$ and $\mathrm{NO}_{2}$ attached with benzene ring, not only the $\mathrm{C}=\mathrm{O}$ can be reduced to $\mathrm{CH}_{2}$, but also the $\mathrm{Cl}$ or $\mathrm{NO}_{2}$ can be reduced to $\mathrm{H}$ or $\mathrm{NH}_{2}$, respectively (entry 10 - 13). For hydrogenation of 2-hydroxy-1-naphthaldehyde (entry 14), the entirely conversion was found and the $\mathrm{C}=\mathrm{O}$ was also transferred to $\mathrm{CH}_{2}$. The more interesting result is that 12 $\%$ of 1-methyl-5,6,7,8-tetrahydronaphthalen-2-ol was obtained besides the main product of 1-methylnaphthalen-2-ol, which indicates that the further hydrogenation was occurred for the condensed nucleus after the $\mathrm{C}=\mathrm{O}$ was transferred to $\mathrm{CH}_{2}$ under this reaction condition. The similar result can be obtained in the hydrogenolysis of benzophenone (entry 15), benzophenone main formed diphenylmethane, and minority (cyclohexylmethyl) benzene was also observed.

The hydrogenolysis experiment of 2-hydroxy-1,2-diphenylethanone was also carried out and gave meaningful result (entry 17), the $\mathrm{C}=\mathrm{O}$ and the $\mathrm{OH}$ were all transferred to $\mathrm{CH}_{2}$, which further confirms the conclusion that $\mathrm{C}=\mathrm{O}$ of aromatic aldehydes or ketones was first reduced to $\mathrm{CHOH}$, and then $\mathrm{CHOH}$ was conversed to $\mathrm{CH}_{2}$ by hydrogenolysis in this reaction system.

\section{Conclusion}

An effectively hydrogenolysis of aromatic aldehydes and ketones was developed. The silica supported Schiff base modified chitosan-palladium catalyst exhibited excellent catalytic ability of aromatic aldehydes or ketones are conversed into corresponding aromatic hydrocarbons in the presence of hydrogen with mild conditions. In this dexoygenation progress, $\mathrm{C}=\mathrm{O}$ of aromatic aldehydes or ketones is firstly converses to $\mathrm{CHOH}$, and then $\mathrm{CHOH}$ is further conversed to $\mathrm{CH}_{2}$. Compared with the comercial 
Table 5. Hydrogenolysis of some different carbonyl compounds catalyzed by $\mathrm{SiO}_{2}$-CS-Schiff-base-Pd catalyst.

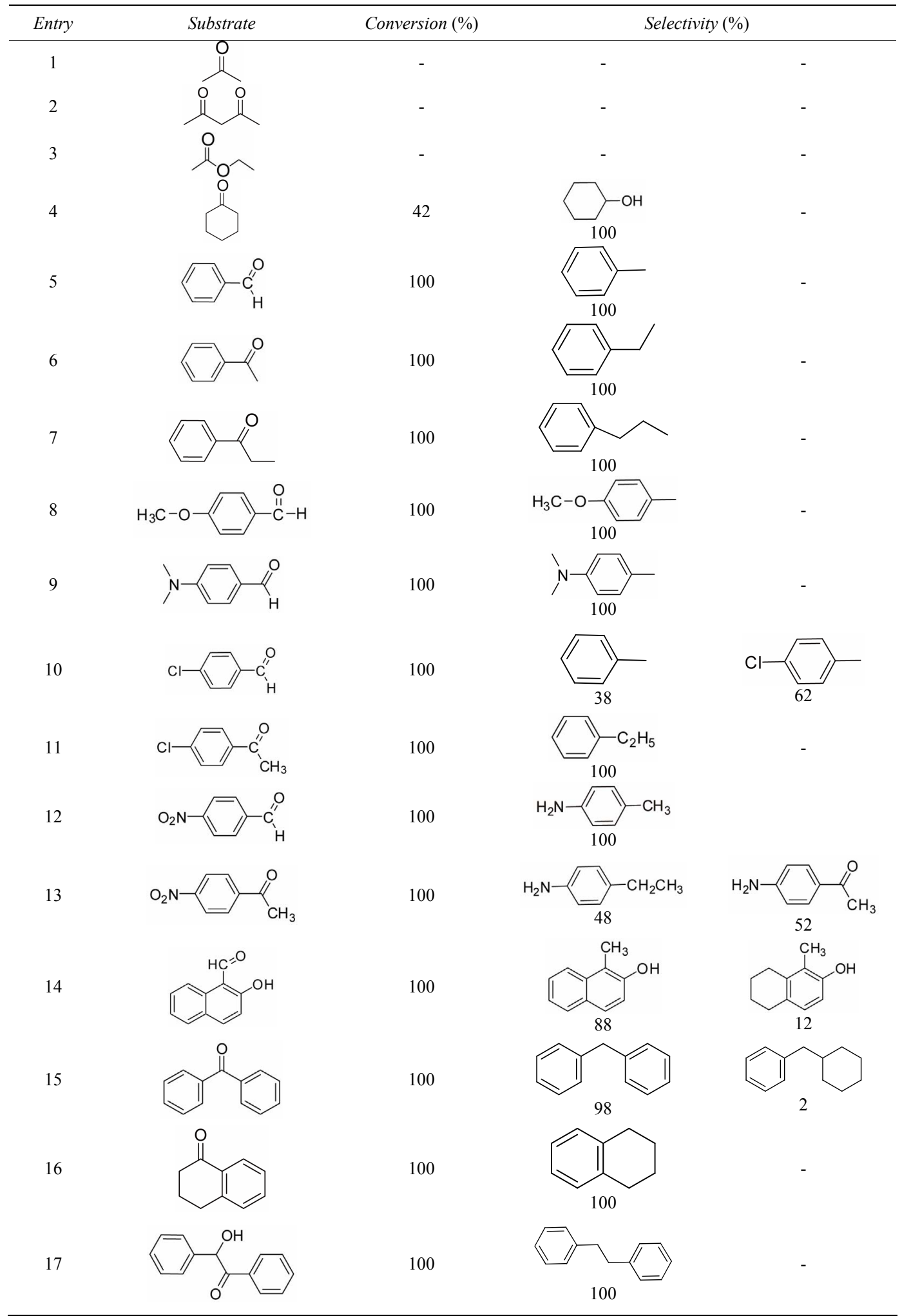

Reaction condition: $0.5 \mathrm{~mL}$ or $0.3 \mathrm{~g}$ substrate, $0.2 \mathrm{~g}$ catalyst, $15 \mathrm{~mL}$ ethanol, $313 \mathrm{~K}, 40 \mathrm{~mL} / \mathrm{min}_{2}, 5 \mathrm{~h}$.

Clemens or Wolff-Kishner reaction, the hydrogenolysis catalyzed by silica supported chitosan-Schiff base-palla- dium catalyst is more eco-friendly, there in no any toxic or corrosive reagents are used. 


\section{Acknowledgements}

The authors are grateful for the support of the Doctor Foundation of Shandong Province (BS2010CL011) and the National Basic Research Program (No.

2010CB234601).

\section{REFERENCES}

[1] N. Desbois, A. Szollosi, A. Maisonial, V. Weber, E. Moreau, J. C. Teulade, O. Chavignon, Y. Blache and J. M. Chezal, "Simple and Convenient Conversion of Acridones into 9-Unsubstituted Acridines via Acridanes using Borane Tetrahydrofuran Complex," Tetrahedron Letters, Vol. 50, No. 49, 2009, pp. 6894-6896. doi:10.1016/j.tetlet.2009.09.141

[2] W. P. Reeves, J. A. Murry, D. W. Willoughby and W. J. Friedrich, "Clemmensen Reductions Using Ultrasonic Irradiation," Synthetic Communications, Vol. 18, No. 1617, 1988, pp. 1961-1966.

[3] C. A. Marques, M. Selva and P. J. Tundo, "Facile Hydrodehalogenation with $\mathrm{H}_{2}$ and $\mathrm{Pd} / \mathrm{C}$ Catalyst under Multiphase Conditions. 3. Selective Removal of Halogen from Functionalized Aryl Ketones. 4. Aryl Halide-Promoted Reduction of Benzyl Alcohols to Alkanes," The Journal of Organic Chemistry, Vol. 60, 1995, pp. 24302435. doi:10.1021/jo00113a024

[4] L. S. Glebov, A. I. Mikaya, A. E. Yatsenko, V. G. Zaikin, G. A. Kliger and S. M. Loktev, "Effective Gas-phase Deoxygenation of Alcohols and Ketones on Iron Catalyst," Tetrahedron Letters, Vol. 26, No. 28, 1985, pp. 33733376. doi:10.1016/S0040-4039(00)98301-1

[5] R. Šuláková, R. Hrdina and G. M. B. Soares, "Oxidation of Azo Textile Soluble Dyes with Hydrogen Peroxide in the Presence of $\mathrm{Cu}(\mathrm{II})$-chitosan Heterogeneous Catalysts," Dyes and Pigments, Vol. 73, No. 1, 2007, pp. 19- 24. doi:10.1016/j.dyepig.2005.10.004

[6] A. Burkhardt, H. Görls and W. Plass, "Nickel(II) Complexes with Schiff-base Ligands Derived from Epimeric Pyranose Backbones as 2,3-Chelators: Modeling the Coordination Chemistry of Chitosan," Carbohydrate Research, Vol. 343, No. 7, 2008, pp. 1266-1277. doi:10.1016/j.carres.2008.01.039

[7] R. Abu-Reziq, D. Avnir and J. Blum, "Catalytic Hydrogenolysis of Aromatic Ketones by a Sol-Gel Entrapped Combined $\mathrm{Pd}-[\mathrm{Rh}(\mathrm{cod}) \mathrm{Cl}]_{2}$ Catalyst," Journal of Molecular Catalysis A: Chemical, Vol. 187, No. 2, 2002, pp. 277-281. doi:10.1016/S1381-1169(02)00235-2

[8] C. Van Doorslaer, J. Wahlen, P. G. N. Mertens, B. Thijs, P. Nockemann, K. Binnemans and D. E. De Vos, "Catalytic Hydrogenolysis of Aromatic Ketones in Mixed Choline-Betainium Ionic Liquids," ChemSusChem, Vol. 1, No. 12, 2008, pp. 997-1005. doi:10.1002/cssc.200800140

[9] S. E. S. Leonhardt, A. Stolle, B. Ondruschka, G. Cravotto, C. De Leo, K. D. Jandt and T. F. Keller, "Chitosan as a Support for Heterogeneous Pd Catalysts in Liquid Phase Catalysis," Applied Catalysis A: General, Vol. 379, No. 1-2, 2010, pp. 30-37. doi:10.1016/j.apcata.2010.02.029

[10] D. Wei, Y. Ye, X. Jia, C. Yuan and W. Qian, "Chitosan as an Active Support for Assembly of Metal Nanoparticles and Application of the Resultant Bioconjugates in Catalysis," Carbohydrate Research, Vol. 345, No. 1, 2010, pp. 74-81. doi:10.1016/j.carres.2009.10.008

[11] N. Sudheesh, S. K. Sharmal and R. S. Shukla, "Chitosan as an Eco-friendly Solid Base Catalyst for the Solventfree Synthesis of Jasminaldehyde," Journal of Molecular Catalysis A: Chemical, Vol. 321, No. 1-2, 2010, pp. 77 82. doi:10.1016/j.molcata.2010.02.005

[12] A. B. Sorokin, F. Quignard, R. Valentin and S. Mangematin, "Chitosan Supported Phthalocyanine Complexes: Bifunctional Catalysts with Basic and Oxidation Active Sites," Applied Catalysis A: General, Vol. 309, No. 2, 2006, pp. 162-168. doi:10.1016/j.apcata.2006.03.060

[13] J. W. Park, M. O. Park and K. K. Park, "Mechanism of Metal ion Binding to Chitosan in Solution. Cooperative Inter- and Intramolecular Chelations," The Bulletin of the Korean Chemical Society, 1984, vol. 5, pp. 108-112.

[14] F. P. Blondet, T. Vincent and E. Guibal, "Hydrogenation of Nitrotoluene Using Palladium Supported on Chitosan Hollow Fiber: Catalyst Characterization and Influence of Operative Parameters Studied by Experimental Design Methodology," International Journal of Biological Macromolecules, Vol. 43, No. 1, 2008, pp. 69-78. doi:10.1016/j.ijbiomac.2007.11.008

[15] F. Peirano, T. Vincent, F. Quignard, M. Robitzer and E. Guibal, "Palladium Supported on Chitosan Hollow Fiber for Nitrotoluene Hydrogenation," Journal of Membrane Science, Vol. 329, No. 1-2, 2009, pp. 30-45.

doi:10.1016/j.memsci.2008.12.022

[16] H. S. Han, S. N. Jiang, M. Y. Huang and Y. Y. Jiang, "Catalytic Hydrogenation of Aromatic Nitro Compounds by Non-Noble Metal Complexes of Chitosan," Polymers for Advanced Technologies, Vol. 7, No. 8, 1996, pp. 704706.

doi:10.1002/(SICI)1099-1581(199608)7:8<704::AID-PA $\underline{\mathrm{T} 567>3.0 . \mathrm{CO} ; 2-3}$

[17] M. Adlim, M. A. Bakar, K. Y. Liew and J. Ismail, "Synthesis of Chitosan-Stabilized Platinum and Palladium Nanoparticles and their Hydrogenation Activity," Journal of Molecular Catalysis A: Chemical, Vol. 212, No. 1-2, 2004, pp. 141-149. doi:10.1016/j.molcata.2003.08.012

[18] T. Vincent, S. Spinelli and E. Guibal, "Chitosan-Supported Palladium Catalyst. II. Chlorophenol Dehalogenation," Industrial \& Engineering Chemistry Research, Vol. 42, No. 24, 2003, pp. 5968-5976. doi:10.1021/ie0301482

[19] M. Y. Yin, G. L. Yuan, Y. Q. Wu, M. Y. Huang and Y. Y. Jiang, "Asymmetric Hydrogenation of Ketones Catalyzed by a Silica-Supported Chitosan-Palladium Complex," Journal of Molecular Catalysis A: Chemical, Vol. 147, No. 1-2, 1999, pp. 93-98. doi:10.1016/S1381-1169(99)00133-8

[20] Y. X. Sun, Y. Guo, Q. Z. Lu, X. L. Meng, X. H. Wu, Y. L. Guo, Y. S. Wang, X. H. Liu and Z. G. Zhang, "Highly Selective Asymmetry Transfer Hydrogenation of Prochiral Acetophenone Catalyzed by Palladium-Chitosan on Silica," Catalsis Letter, Vol. 100, No. 3-4, 2005, pp. $213-$ 217. doi:10.1007/s10562-004-3458-1

[21] J. E. dos Santos, E. R. Dockal and É. T. G. Cavalheiro, 
"Synthesis and Characterization of Schiff Bases from Chitosan and Salicylaldehyde Derivatives," Carbohydrate Polymers, Vol. 60, No. 3, 2005, pp. 277-282. doi:10.1016/i.carbpol.2004.12.008

[22] G. K. Moore and G. A. F. Roberts, "A Compound Hydrodynamic Shape Function Derived from Viscosity and Molecular Covolume Measurements," International Journal of Biological Macromolecules, Vol. 3, No. 5, 1981, pp. 337-341. doi:10.1016/0141-8130(81)90053-2

[23] S. W. Gong, H. F. He, C. Q. Zhao, L. J. Liu and Q. X. Cui, "Convenient Deoxygenation of Aromatic Ketones by Silica Supported Chitosan Schiff-Base Palladium Catalyst," Synthetic Communications, Vol. 42, No. 4, 2012, pp.
574- 581. doi:10.1080/00397911.2010.527423

[24] J. Tong, Z. Li and C. Xia, "Highly Efficient Catalysts of Chitosan-Schiff Base Co(II) and Pd(II) Complexes for Aerobic Oxidation of Cyclohexane in the Absence of Reductants and Solvents," Journal of Molecular Catalysis A: Chemical, Vol. 231, No. 1-2, 2005, pp. 197-203.

doi:10.1016/j.molcata.2005.01.011

[25] H. F. He, S. W. Gong, L. J. Liu and Q. X.Cui and H.D. Yin, "Hydrogenation of Aromatic Ketones to Aromatic Hydrocarbons over $\mathrm{SiO}_{2}$-Supported Chitosan Schiff-Base Palladium Catalyst," Chinese Journal of Catalysis, Vol. 31, No. 7, 2010, pp. 846-850. 